\title{
Postoperative Pain Therapy with Laser Acupuncture after Cesarean Section under Spinal Anesthesia: A Double-Blinded, Randomized, Placebo-Controlled Trial
}

\author{
Annika Brase ${ }^{a}$ Dominik Brauchle ${ }^{a}$ Azzeddine Kermad ${ }^{a}$ Thomas Volk ${ }^{a}$ \\ Emanuela Morinello $^{a}$ Sven Gottschling ${ }^{b}$ Sven Oliver Schneider ${ }^{a}$ \\ aDepartment of Anesthesiology, Intensive Care Medicine and Pain Medicine, Saarland University Hospital Medical

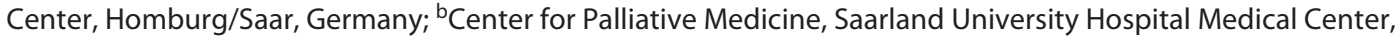 \\ Homburg/Saar, Germany
}

\section{Keywords}

Pain therapy · Cesarean section · Laser acupuncture

\begin{abstract}
Purpose: The aim of our prospective, randomized, doubleblind, placebo-controlled study was to investigate the effect of laser acupuncture on postoperative pain in women undergoing cesarean section. Methods: 99 women (mean age $32 \pm 5$ years) scheduled for cesarean section under spinal anesthesia at Saarland University Hospital Medical Center, Homburg (Germany) were enrolled in this trial after giving written consent. Patients were randomized in two groups receiving a course of 3 treatments over 3 days with either active or placebo laser. Each acupuncture session treated Di-4 and Shen-men of both hands and ears. Primary outcome was defined as the pain severity at rest measured by Numeric Rating Scale (NRS) on the first postoperative day. Secondary outcome measures included analgesic consumption, time to mobilization, and length of stay. Treatment occurred on the operation day and on the following 2 days. Data were collected by a standardized questionnaire. Results: From 95 included patients, 80 were analyzed. No statistical difference between both groups were observed for pain severity at rest on the first postoperative day after cesarean section (MannWhitney $\mathrm{U}$ test, $p=0.850$, verum group [mean \pm standard deviation]: $3.3 \pm 2.1$ vs. placebo group: $3.2 \pm 1.9$ ). Secondary outcome measures regarding analgesic consumption
\end{abstract}

showed no difference in NSAID or opioid between treatment and placebo group. Laser acupuncture showed no effect on time to mobilization and length of stay. Conclusion: Our study could not demonstrate significant effects by the application of laser acupuncture on postoperative pain in women undergoing cesarean section.

(c) 2022 The Author(s).

Published by S. Karger AG, Basel

\section{Postoperative Schmerztherapie mittels Laserakupunktur nach Sectio Cesarea unter Spinalanästhesie: Eine doppelblinde, randomisierte, placebokontrollierte Studie}

\section{Schlüsselwörter}

Laserakupunktur · Sectio Cesarea · Schmerztherapie

\section{Zusammenfassung}

Zielsetzung: Das Ziel unserer prospektiven, randomisierten, placebokontrollierten Studie ist es, den Effekt von Laserakupunktur auf die Schmerztherapie von Frauen nach Kaiserschnitt unter Spinalanästhesie zu untersuchen. Methoden: 99 Frauen (Alter $32 \pm 5$ Jahre), die eine Sectio Cesarea in Spinalanästhesie am Universitätsklinikum des Saarlandes (Homburg, Deutschland) bekommen haben, wurden nach schriftlicher und mündlicher

\section{(C) 2022 The Author(s).}

Published by S. Karger AG, Basel

This article is licensed under the Creative Commons Attribution 4.0 International License (CC BY) (http://www.karger.com/Services/ OpenAccessLicense). Usage, derivative works and distribution are permitted provided that proper credit is given to the author and the original publisher.
Correspondence to:

Azzeddine Kermad, kermad@ outlook.de 
Aufklärung und Einwilligung für unsere Studie rekrutiert. Die Patientinnen wurden in zwei Gruppen randomisiert und wurden täglich für drei Tage entweder mit einem aktiven oder einem Placebo Laser behandelt. Jede Anwendung umfasste die Stimulation von Di-4 sowie Shen-men an beiden Händen und Ohren. Als primärer Outcome wurde die Schmerzintensität in Ruhe am ersten postoperativen Tag definiert. Diese wurde mittels der Numeric Rating Scale (NRS) ermittelt. Als sekundäre Outcomeparameter wurden der Analgetikaverbrauch, die Mobilisation und die Krankenhausverweildauer analysiert. Die Behandlungen erfolgten am Tag der OP und an den ersten zwei postoperativen Tagen. Die Daten wurden mittels Fragebögen erhoben. Ergebnisse: 95 Patientinnen wurden in die Studie eingeschlossen, davon wurden 80 statistisch analysiert. Es zeigte sich kein statistisch signifikanter Unterschied zwischen den beiden Gruppen in der Schmerzintensität in Ruhe am ersten postoperativer Tag nach dem Kaiserschnitt (Mann-Whitney U-Test, $p=0,850$, Verum-Gruppe [Mittelwert \pm Standardabweichung]: 3,3 \pm 2,1 vs. Placebo-Gruppe: 3,2 $\pm 1,9$ ). Auch die sekundären Outcomeparameter zeigten keinen signifikanten Unterschied. Fazit: Unsere Studie zeigte keinen signifikanten Effekt von aktiver Laserakupunktur für die Schmerztherapie von Patientinnen nach Kaiserschnitt.

C 2022 The Author(s).

Published by S. Karger AG, Basel

\section{Introduction}

In Germany, the cesarian section rate is approximately $35 \%$ [1] and is associated with moderate to severe pain after surgery. The first-line therapy for pain control consists of systemic application of analgesics and the use of regional analgesia $[2,3]$.

In the early phase after cesarean section there are various difficulties in the use of pain medication [4]. On the one hand, the medication should not influence breastfeeding and not affect the recovery and consciousness of the mother. On the other hand, cesarean section is a moderate to severe painful procedure necessitating adequate analgesia. Compared to other gynecological surgeries cesarean sections are associated with significantly higher pain intensity and higher request for additional analgesics [4].

Therefore, the application of alternative methods to reduce pain in women after cesarean section should be considered. Various approaches for drug-free pain control such as relaxation techniques, acupuncture, and meditation may be a useful method to additionally treat pain [5-11].

Although the number of clinical trials has been constantly rising in the last years, getting such unconventional approaches into evidence-based medicine is often com- plex. The effects of auricular and whole body needle acupuncture for postoperative pain control after cesarean section obtained promising results $[12,13]$.

Considering the unclear effectivity of laser acupuncture on pain after cesarean section, we planned a prospective, randomized, placebo-controlled double-blinded trial to investigate whether a highly standardized active laser acupuncture protocol is superior to placebo laser acupuncture for reducing postoperative pain intensity after cesarean section.

\section{Materials and Methods}

This study was approved by the ethical committee (Ärztekammer des Saarlandes, reference number 133/17), and written informed consent was obtained from all patients participating in the trial. The trial was registered at drks.de (DRKS00021519) on March 16, 2021. Patients were informed in detail about the doubleblinded and placebo-controlled study design during the preoperative information talk with the patients which occurred at least 24 $\mathrm{h}$ before the cesarean section. After receiving informed consent for the surgery and the spinal anesthesia they were informed about the trial orally and in written form. Participation was voluntary and withdrawal was possible at any time without giving a reason. Our patients were recruited in the Department for Obstetrics of the Saarland University Hospital Medical Center between April 2018 and January 2019.

Inclusion criteria were age over 18 years, ASA score (American Society of Anesthesiologists) <III, and planned spinal anesthesia for the cesarean section. Exclusion criteria were age under 18 years, ASA score >III, general anesthesia, refusal to participate, inability to speak German or to communicate otherwise, history of drug abuse, duration of surgery over $60 \mathrm{~min}$, or the presence of relevant intraoperative complications.

After study inclusion, patients were randomized either in group A or in group B by a computerized list and stated which laser (A or B) was going to be used. Sample size was calculated using the pwr package version 1.2-1 in R version 3.4.0. Block randomization was performed using the randomize $\mathrm{R}$ package version 1.4 in $\mathrm{R}$ version 3.4.0. The list was balanced to get equal amounts in each group.

The laser devices were optically identical and labeled with "A" or "B." The display on both lasers showed exactly the same content, both engines emitted the same red guiding light and the same sound but only one of the lasers emitted an invisible laser beam. After recruitment was stopped in August 2019 the study was deblinded. Laser " $A$ " turned out to be the verum laser, laser " $B$ " the placebo laser. The active laser was a class $3 \mathrm{~B}$ laser. The device used was a schwa-medico Modulas-Handy 2/99, $30 \mathrm{~mW}, 830 \mathrm{~nm}$, continuous wave, power density $3.8 \mathrm{~W} / \mathrm{cm}^{2}$ with $1 \mathrm{~mm}$ laser beam diameter (REF 205237, schwa-medico, Ehringshausen, Germany). During the laser procedure the patient, the acupuncturist, and any other person in the room wore protective glasses.

Both laser devices - verum and placebo - appeared to be identical. Thus, the patient and the acupuncturist were unable to differentiate if the treatment was done with the active or the placebo laser device.

We chose to use a highly standardized laser acupuncture protocol to obtain a better comparability with other studies. As shown in Figure 1 the observation period consisted of 4 days: the day before the cesarean section, the operation day, the first and second 


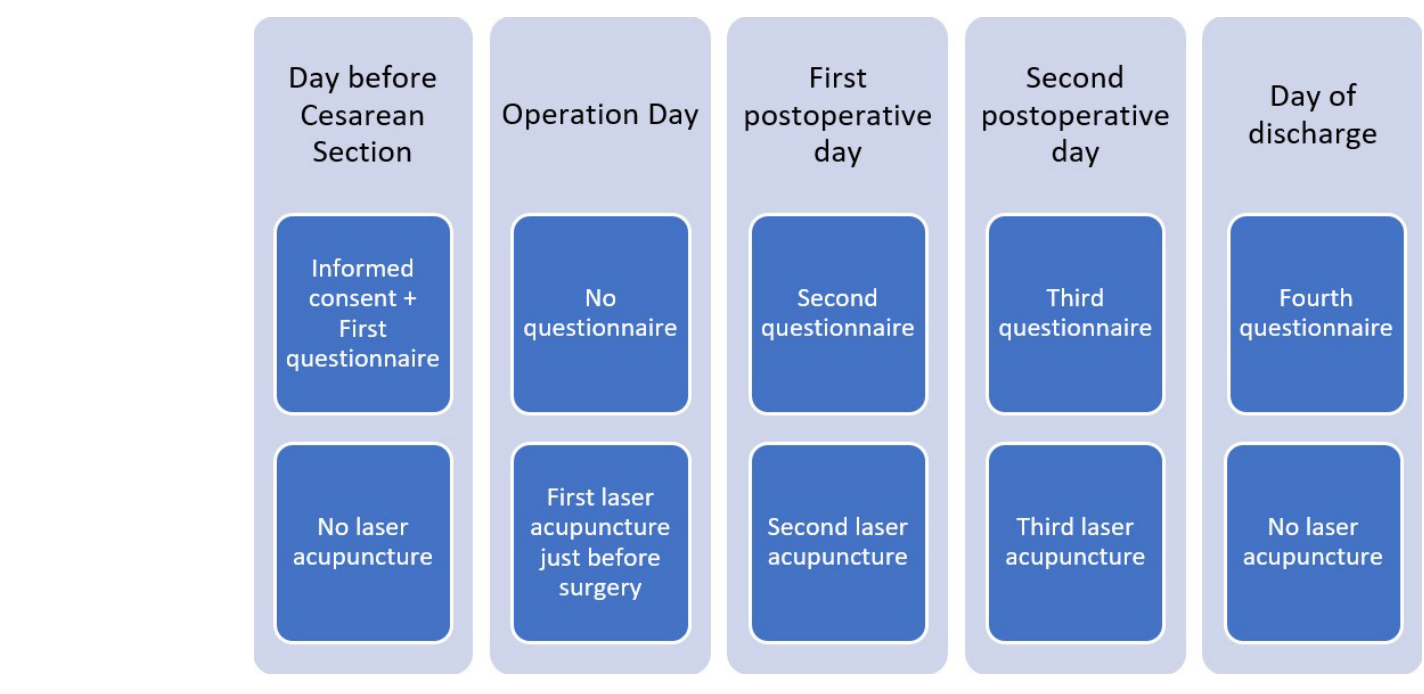

Fig. 1. Patients' timeline.

postoperative days, and the day of discharge. Each patient received laser acupuncture three times: just before getting in the operation room, on the first postoperative day and on the second postoperative day (Fig. 1).

\section{Laser Acupuncture Protocol}

During every session, two acupuncture points, Di-4 and ShenMen of the ear, were stimulated on the right and on the left side, each for $1 \mathrm{~min}$, keeping vertical contact to the skin. Every patient was handed a questionnaire 1 day before the surgery, on the first and second postoperative day after receiving the laser acupuncture and on the day of discharge just before leaving the hospital. We designed the questionnaire especially for this study according to the International Association for the Study of Pain (IASP) questionnaires. The choice of the acupuncture points occurred with the help of Dr. Patric Bialas and Prof. Dr. Sven Gottschling, who both practice acupuncture on a regular basis. Prof. Gottschling is the head of palliative care unit in our hospital. He practices acupuncture on palliative patients as well as on non-palliative patients for pain therapy on a regular basis. He owns the "A-Diplom" of the "Deutsche Akademie für Akupunktur" and completed the additional training with $500 \mathrm{~h}$ of training. Dr. Patric Bialas is a supervisor anesthesiologist in our department. He was in charge of the pain care unit for many years and has now a private practice focusing exclusively on pain therapy. He has the "A-Diplom" of the "Deutsche Akademie für Akupunktur."

\section{Assessment of Pain Severity}

Pain was measured using a Numeric Rating Scale (NRS; 0-10) $[14,15]$. We asked patients for abdominal pain intensity at rest and maximal pain during the observation period. The patients completed the questionnaire just after receiving the laser acupuncture.

\section{Analgesic Consumption}

Baseline pain treatment was provided with NSAIDs and opioids by gynecologists and was set at the discretion of the physician. Several kind of NSAIDs and weak opioids were administered, depending on the patients' needs. We documented the consumption of pain medication on each day. NSAIDs were summed up and statistical analysis was done considering the amount of pills each patient received per day. Opioid doses were converted in morphine equivalents. Tramadol was converted with the factor 0.1 , oxycodone with factor 2 , piritramide with 0.7 , and pethidine with 0.2 . Furthermore, we investigated the patient's mobility and deter- mined length of the hospital stay. To examine patient's mobility we asked for an overall self-consideration on each day via questionnaires. Patients could choose between five options: "I can stand up, walk, and carry my baby," "I can stand up and walk a few steps, for example to the toilet," "I can stand up but need to sit down shortly after," "I can sit in bed," "I can barely move."

\section{Data Evaluation and Statistical Analysis}

Data analyses were conducted by using SPSS (SPSS Statistics 24, IBM, Armonk, USA). Categorical variables are presented as numbers (percentages). Continuous variables are expressed as mean \pm standard deviation (SD) or as median [interquartile range] if not normally distributed. Data were assessed for normality using a Kolmogorov-Smirnov test. The frequencies were compared using a Fischer's exact test, parametrical data using a $t$ test, and nonparametrical data using a Mann-Whitney U test. Significance level was set at $p<0.005$.

The primary outcome, pain intensity at rest on the first postoperative day, was compared in group verum and placebo (respectively A and B) using Fischer's exact test.

Secondary outcomes were the consumption of pain medication such as NSAIDs and opioids, pain values on the second postoperative day and the day of discharge and the extent of mobilization and length of stay. Those results were compared in group verum and placebo using a Mann-Whitney U test.

\section{Sample Size Determination}

A difference of at least 1.5 on the NRS was defined as a significant change. To detect this difference a sample size of 40 patients per group was calculated with a power of $80 \%$ and a type-I error of $5 \%$. Power calculation was based on a similar needle acupuncture pilot trial by Hesse et al. [16].

\section{Results}

After written consent, 99 women participated in our study. Four patients dropped out before starting the trial giving childbirth before the planned cesarean section date. 95 patients were randomized in verum or placebo group. 10 patients of the verum and 5 patients of the placebo group dropped out secondarily due to protocol vio- 
Table 1. Patients' baseline characteristics

\begin{tabular}{llll}
\hline Parameter (mean/amount) & Verum $\left(n_{V}=38\right)$ & Placebo $\left(n_{\mathrm{P}}=42\right)$ & $p$ value \\
\hline Age, years & $32 \pm 5$ & $32 \pm 5$ & 0.511 \\
BMI & $30.7 \pm 6.2$ & $32.5 \pm 6.5$ & 0.214 \\
OP time, min & $41.2 \pm 9.9$ & $37.98 \pm 11.7$ & 0.184 \\
ASA score & $8(21 \%)$ & $8(19 \%)$ & 0.809 \\
$\quad$ ASA I & $27(71.1 \%)$ & $32(76.2 \%)$ & \\
$\quad$ ASA II & $3(7.9 \%)$ & $2(4.8 \%)$ & \\
$\quad$ ASA III & & & \\
Re-cesarean section & $18(47.4 \%)$ & $18(42.9 \%)$ & 0.382 \\
$\quad$ Yes & $20(52.6 \%)$ & $24(57.1 \%)$ & \\
$\quad$ No & $11(28.9 \%)$ & $10(23.8 \%)$ & 0.602 \\
Patient uses alternative medicine in daily life & & \\
$\quad$ Yes & $27(71.1 \%)$ & $32(76.2 \%)$ & \\
$\quad$ No & & & \\
Patient expects that...: & $1(2.6 \%)$ & $3(7.2 \%)$ & 0.147 \\
$\quad$ Acupuncture does not work & $19(50 \%)$ & $20(47.6 \%)$ & \\
$\quad$ Acupuncture may work but never tried & $11(26.2 \%)$ & \\
$\quad$ Acupuncture may work and already tried & $16(42.1 \%)$ & $8(19 \%)$ & \\
$\quad$ Acupuncture works very good & $2(5.3 \%)$ & & \\
\hline
\end{tabular}

BMI, body mass index; OP time, operation time; C-section, cesarean section.

Fig. 2. Flowchart.

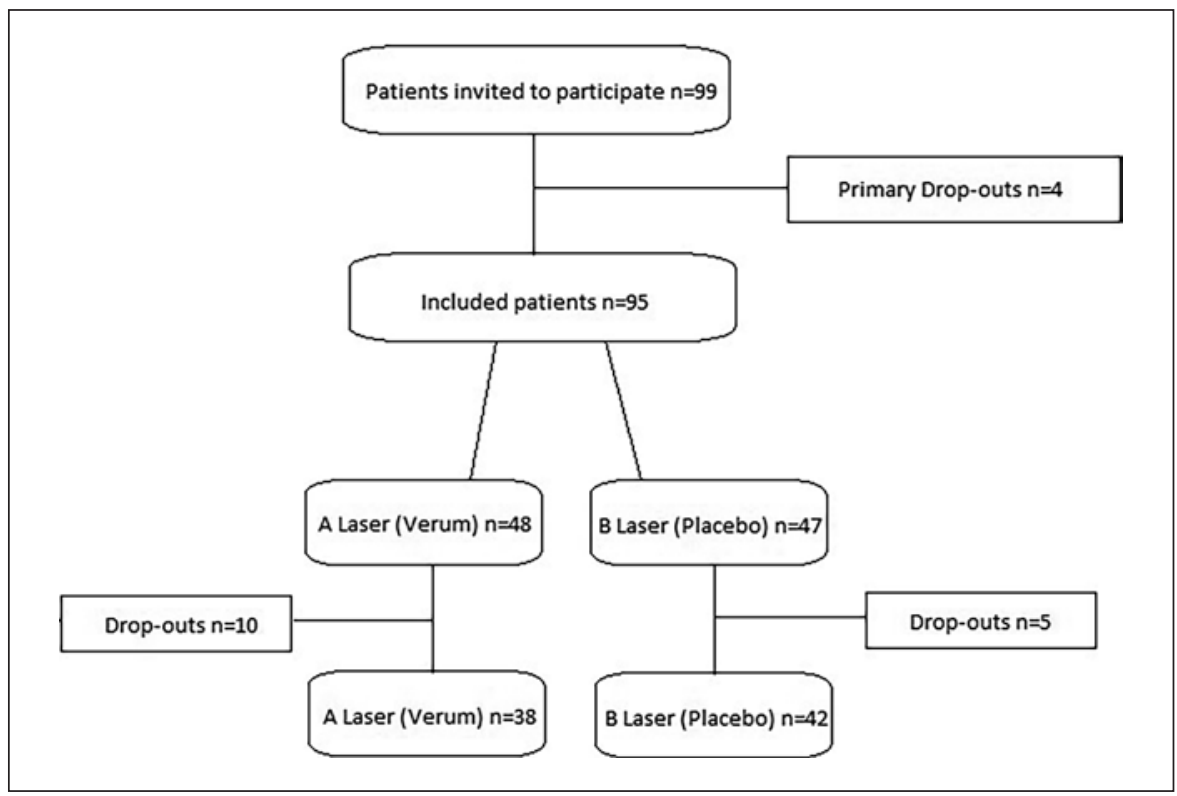

lation, resulting in two groups with 38 (verum) and 42 (placebo) patients (Fig. 2). Only patients who completed the intervention and answered each questionnaire were included in statistical analysis. No patient reported side effects or wanted to interrupt the treatment, prematurely.

The patients' characteristics are summarized in Table 1 . Verum includes the patients treated with the verum laser and placebo includes the patients treated with placebo laser. There were no statistically significant differences between the two groups.

Results for pain in rest, at movement and maximal pain on the first postoperative day are summarized in $\mathrm{Ta}$ - ble 2 . There was no statistically significant difference between verum and placebo group.

The descriptive statistics regarding pain on the second postoperative day and on the day of discharge are summarized in Table 3 and 4 . There was no statistically significant difference between verum and placebo group.

There was no statistically significant difference in perioperative opioid or NSAIDs consumption between verum and placebo group. There was no statistically significant difference in time to mobilization and length of stay between the verum and the placebo group (Table 5). 
Table 2. Pain on the first postoperative day

\begin{tabular}{lllll}
\hline & & $\begin{array}{l}\text { Verum } \\
\left(n_{\mathrm{V}}=38\right)\end{array}$ & $\begin{array}{l}\text { Placebo } \\
\left(n_{\mathrm{p}}=42\right)\end{array}$ & $p$ value \\
\hline Pain at rest & Median & 3 & 3 & 0.852 \\
& Min & 0 & 0 & \\
& Max & 8 & 7 & \\
& IQR & 3 & 3 & \\
\hline Pain during & Median & 6 & 5 & 0.154 \\
movement & Min & 1 & 1 & \\
& Max & 9 & 10 & \\
& IQR & 3 & 3 & 0.959 \\
Maximum pain & Median & 7 & 7 & \\
& Min & 1 & 2 & \\
& Max & 10 & 10 & \\
& IQR & 3 & 3 & \\
\hline
\end{tabular}

Data were compared using Fischer's exact test.

Table 4. Pain on the day of discharge

\begin{tabular}{lllll}
\hline & & $\begin{array}{l}\text { Verum } \\
\left(n_{\mathrm{V}}=38\right)\end{array}$ & $\begin{array}{l}\text { Placebo } \\
\left(n_{\mathrm{P}}=42\right)\end{array}$ & $p$ value \\
\hline Pain at rest & Median & 1 & 0.5 & 0.230 \\
& Min & 0 & 0 & \\
& Max & 5 & 4 & \\
\hline Pain during & IQR & 2 & 1 & 0.039 \\
movement & Median & 3 & 2 & \\
& Min & 0 & 0 & 0.168 \\
& Max & 7 & 9 & \\
\hline Maximum pain & Median & 2 & 2 & \\
& Min & 1 & 3 & \\
& Max & 10 & 8 & \\
& IQR & 3 & 2 & \\
\hline
\end{tabular}

Data were compared using Fischer's exact test.

\section{Discussion}

In this double-blinded, randomized, placebo-controlled trial, we found no effect of active laser acupuncture on postoperative pain after cesarean section under spinal anesthesia.

Treating patients with acupuncture for postoperative pain is not a standard procedure at our hospital. Therefore, one goal of our group was to develop a highly standardized protocol which is simple in its application.

Both considered Di- 4 and Shen-Men of the ear as reliable and easy to locate points and appropriate to relieve the postoperative pain. In addition to our experts' opinion we did literature research and found several trials suggesting the use of Di- 4 and Shen-Men of the ear to alleviate postoperative pain
Table 3. Pain on the second postoperative day

\begin{tabular}{lllll}
\hline & & $\begin{array}{l}\text { Verum } \\
\left(n_{\mathrm{V}}=38\right)\end{array}$ & $\begin{array}{l}\text { Placebo } \\
\left(n_{\mathrm{P}}=42\right)\end{array}$ & $p$ value \\
\hline Pain at rest & Median & 2 & 1 & 0.531 \\
& Min & 0 & 0 & \\
& Max & 9 & 9 & \\
\hline Pain during & QQR & 1 & 2 & 0.133 \\
movement & Median & 3 & 3 & \\
& Min & 0 & 0 & \\
& Max & 8 & 9 & 0.423 \\
& IQR & 1 & 2 & \\
\hline Maximum pain & Median & 5 & 4 & \\
& Min & 2 & 0 & \\
& Max & 10 & 9 & \\
& IQR & 2 & 2 & \\
\hline
\end{tabular}

Data were compared using Fisher's exact test.

Table 5. Length of stay

\begin{tabular}{lllll}
\hline & & $\begin{array}{l}\text { Verum } \\
\left(n_{\mathrm{V}}=38\right)\end{array}$ & $\begin{array}{l}\text { Placebo } \\
\left(n_{\mathrm{P}}=42\right)\end{array}$ & $p$ value \\
\hline Length of stay, & Median & 4 & 3 & 0.162 \\
days & Min & 2 & 2 & \\
& Max & 6 & 5 & \\
& IQR & 1 & 1 & \\
\hline
\end{tabular}

Data were compared using Mann-Whitney U test.

$[12,13,17-19]$. A meta-analysis investigating the effectivity of different acupuncture points in reducing postoperative pain therapy suggested the use of Di-4 [20]. A similar trial reported the application of ear acupuncture against acute pain after cesarean section, abdominal and cardiac surgery. Many of the studies included Shen-Men point of the ear to achieve pain reduction [21]. A direct comparison to other laser acupuncture trials is not possible to this date because we could not find studies that investigated the effect of laser acupuncture on pain after cesarean section so that comparison of results is not really reliable. In general, laser acupuncture was well tolerated in our study supporting the results of other published trials investigating laser acupuncture [9-11]. We mainly preferred laser acupuncture over needle acupuncture due to practical reasons. A double-blinded randomized trial seemed to be nearly impossible with needle acupuncture.

The postoperative pain after cesarean section reaches its worst during the first $24 \mathrm{~h}$ after the surgery [4]; therefore, we set our primary outcome measure on the pain in rest on the first postoperative day. Our treatment with active laser acupuncture did not achieve neither a clinical nor a statistically significant improvement of pain levels. Pain levels fall drastically throughout the days after the surgical procedure but as 
shown from our statistical analysis there was no difference between the use of active and placebo laser acupuncture.

Pain clearly influences the patients' mobility - the lower the pain, the better the mobility. If we consider that women undergoing cesarean section are primarily healthy and young it is alarming to see that over one third of them could barely stand on the first postoperative day. It is therefore of crucial importance to find applicable ways to improve postoperative pain after cesarean section, in order to allow the young mothers to take care of their children and not to stay stuck in the "patient role." At our hospital, women normally discharge on the third postoperative day after cesarean section if there is no noticeable complication and the patient is fine. This was also applicable and independently from stimulation with active or placebo laser acupuncture, no significant difference was recorded.

In this study we had mainly three limitations. One certainly was the non-standardized pain medication which made statistical analysis complex. A validated and practicable method to achieve NSAID comparability is still missing even though there have been some trials on this subject as for example the Oxford League Table [22].

Second, the choice of laser acupuncture versus needle acupuncture could have mitigated the effect of the treatment. The typical deqi sensation described by the patient during needle acupuncture is often considered as essential to achieve the goal of the treatment. Deqi is described as a spreading, numb, and heavy feeling during the insertion of an acupuncture needle in an acupuncture point. The deqi sensation has been analyzed in different clinical trials [23]. It is assumed, that the analgesic effect of acupuncture derives from a $\mathrm{C}$-fiber stimulation which leads to a decrease in pain transmission. C-fiber may also be involved in the deqi sensation [23]. Though laser acupuncture shows the same activation in functional magnet resonance imaging (fMRI) as needle acupuncture often the deqi sensation is missed by the patient [24]. This could be a reason why our treatment showed no significant pain-reducing effect.

As third limitation, we might have chosen our primary outcome measure too strictly and too focused on pain values. As we could see in the results section patients showed a very high acceptance to laser acupuncture even without experiencing a statistically significant benefit from it. Maybe if we expanded our outcome measures to recovery scores there would have been a more complete picture of the effects of laser acupuncture on postoperative pain.

\section{Conclusion}

No significant statistical difference was observed between the use of active laser acupuncture and placebo laser acupuncture in relieving postoperative pain after cesarean section under spinal anesthesia.

\section{Statement of Ethics}

This study was approved by the ethical committee (Ärztekammer des Saarlandes, reference number 133/17), and written informed consent was obtained from all patients participating in the trial. The trial was registered at drks.de (DRKS00021519).

\section{Conflict of Interest Statement}

Annika Brase: the presented data are part of her doctoral thesis. Domnik Brauchle: none. Azzeddine Kermad: none. Thomas Volk: none. Emanuela Morinello: none. Sven Gottschling: none. Sven Oliver Schneider: none.

\section{Funding Sources}

This research did not receive any specific grant from funding agencies in the public, commercial, or not-for-profit sectors.

\section{Author Contributions}

Annika Brase: this author made substantial contributions to the concept and the design of the study; the acquisition, the analysis, and the interpretation of the data; drafting the article; and revising it critically for important intellectual content. Domnik Brauchle: this author made substantial contributions to the concept and the design of the study; the acquisition, the analysis, and the interpretation of the data; drafting the article; and revising it critically for important intellectual content. Azzeddine Kermad: this author made substantial contributions to the concept and the design of the study; the acquisition, the analysis, and the interpretation of the data; drafting the article; and revising it critically for important intellectual content. Thomas Volk: this author made substantial contributions to the concept and the design of the study; the acquisition, the analysis, and the interpretation of the data; drafting the article; and revising it critically for important intellectual content. Emanuela Morinello: this author made substantial contributions to the concept and the design of the study; the acquisition, the analysis, and the interpretation of the data; drafting the article; and revising it critically for important intellectual content. Sven Gottschling: this author made substantial contributions to the concept and the design of the study; the acquisition, the analysis, and the interpretation of the data; drafting the article; and revising it critically for important intellectual content. Sven Oliver Schneider: this author made substantial contributions to the concept and the design of the study; the acquisition, the analysis, and the interpretation of the data; drafting the article; and revising it critically for important intellectual content.

\section{Data Availability Statement}

All data generated or analyzed during this study are included in this article. Further enquiries can be directed to the corresponding author. 


\section{References}

1 Press release \#349 from 17 September 2018. $30.5 \%$ of hospital deliveries by Caesarean section in 2017 Destatis, Statistisches Bundesamt. Available from: https://www.destatis.de/ EN/Press/2018/09/PE18_349_231.html;jsessi onid $=6318$ EAF58D87331C96DBCDC29F16 4D7F.live712.other (last visit 2021 Jun 29).

2 Bremerich D, Annecke T, Chappell D. German S1 guidelines "obstetric analgesia and anesthesia”. Die geburtshilfliche Analgesie und Anästhesie. S1-Leitlinie der deutschen gesellschaft für anästhesiologie und intensivmedizin in zusammenarbeit mit der deutschen gesellschaft für gynäkologie und geburtshilfe. 2020:1-38.

3 Bremerich D, Annecke T, Chappell D. German S1 guidelines "Obstetric analgesia and anesthesia". Die geburtshilfliche Analgesie und Anästhesie. S1-Leitlinie der deutschen gesellschaft für anästhesiologie und intensivmedizin in zusammenarbeit mit der deutschen gesellschaft für gynäkologie und geburtshilfe; 2020 Jun. (last visit 2021 Jun 29). Available from: https: //www.awmf.org/uploads/tx szleitlinien/001-0381_S1_Die-geburtshilfliche-Analgesie-und-Anaesthesie_2020-03. pdf.other.

4 Marcus H, Gerbershagen HJ, Peelen LM, Aduckathil S, Kappen TH, Kalkman CJ, et al. Quality of pain treatment after caesarean section: results of a multicentre cohort study. Eur J Pain. 2015 Aug;19(7):929-39.

5 Landau R. Post-cesarean delivery pain. Management of the opioid-dependent patient before, during and after cesarean delivery. Int J Obstet Anesth. 2019;39:105-16.

6 Zimpel SA, Torloni MR, Porfirio GJ. Complementary and alternative therapies for postcaesarean pain. Cochrane Database Syst Rev. 2020;9:CD011216.

7 Baxter GD, Bleakley C, McDonough S. Clinical effectiveness of laser acupuncture: a systematic review. J Acupunct Meridian Stud. 2008;1(2):65-82.
$8 \mathrm{Wu}$ MS, Chen KH, Chen IF. The efficacy of acupuncture in post-operative pain management: a systematic review. PLoS One. 2016; 11:e0150367.

9 Wylde V, Dennis J, Beswick AD, Bruce J, Eccleston C, Howells N, et al. Systematic review of management of chronic pain after surgery. Br J Surg. 2017;104:1293-306.

10 Tedesco D, Gori D, Desai KR, Asch S, Carroll IR, Curtin C, et al. Drug-free interventions to reduce pain or opioid consumption after total knee arthroplasty: a systematic review and meta-analysis. JAMA Surg. 2017; 152: e172872.

11 Quinlan-Woodward J, Gode A, Dusek JA, Reinstein AS, Johnson JR, Sendelbach S. Assessing the impact of acupuncture on pain, nausea, anxiety, and coping in women undergoing a mastectomy. Oncol Nurs Forum. 2016; 43:725-32.

12 Liu XL, Tan JY, Molassiotis A, Suen LK, Shi Y. Acupuncture-point stimulation for postoperative pain control: a systematic review and meta-analysis of randomized controlled trials. Evid Based Complement Alternat Med. 2015;2015:657809.

13 Kim M, Moss D, Crawford P. Battlefield acupuncture for post-partum pain: a randomized controlled trial. Explore. 2019;15:409-14.

14 Ferraz MB, Quaresma MR, Aquino LR, Atra E, Tugwell P, Goldsmith CH. Reliability of pain scales in the assessment of literate and illiterate patients with rheumatoid arthritis. J Rheumatol. 1990 Aug;17(8):1022-4.

15 Hawker GA, Mian S, Kendzerska T, French M. Measures of adult pain: visual analog scale for pain (VAS Pain), numeric rating scale for pain (NRS Pain), McGill pain questionnaire (MPQ), short-form mcgill pain questionnaire (SF-MPQ), chronic pain grade scale (CPGS), short form-36 bodily pain scale (SF-36 BPS), and measure of intermittent and constant osteoarthritis pain (ICOAP). Arthritis Care Res. 2011 Nov;63 Suppl 11:S240-52.
16 Hesse T, Henkel B, Zygmunt M, Mustea A, Usichenko TI. Acupuncture for pain control after Caesarean section: a prospective observational pilot study. Acupunct Med. 2016;34: 14-9.

17 Jaić KK, Turković TM, Pešić M, Djaković I, Košec V, Košec A. Auricular acupuncture as effective pain relief after episiotomy: a randomized controlled pilot study. Arch Gynecol Obstet. 2019 Nov;300(5):1295-301.

18 Lin JG, Lo MW, Wen YR, Hsieh CL, Tsai SK, Sun WZ. The effect of high and low frequency electroacupuncture in pain after lower abdominal surgery. Pain. 2002;99:509-14.

19 Oates A, Benedict KA, Sun K, Brakeman PR, Lim J, Kim C. Laser acupuncture reduces pain in pediatric kidney biopsies: a randomized controlled trial. Pain. 2017;158:103-9.

20 Jan AL, Aldridge ES, Rogers IR, Visser EJ, Bulsara MK, Niemtzow RC. Does ear acupuncture have a role for pain relief in the emergency setting? A systematic review and meta-analysis. Med Acupunct. 2017;29:27689.

21 Gottschling S, Meyer S, Gribova I, Distler L, Berrang J, Gortner L, et al. Laser acupuncture in children with headache: a double-blind, randomized, bicenter, placebo-controlled trial. Pain. 2008;137:405-12.

22 Bandolier. Acute pain: the 2007 Oxford league table of analgesic efficacy. 2007. Available from: http://www.bandolier.org.uk/ booth/painpag/Acutrev/Analgesics/lftab. html.other.

23 Salih N, Bäumler PI, Simang M, Irnich D. Deqi sensations without cutaneous sensory input: results of an RCT. BMC Complement Altern Med. 2010;10:81.

24 Siedentopf CM, Haala IA, Koppelstätter F, Verius M, Golaszewski S, Schikora D, et al. Placebo-laser kontrollierte, computer gesteuerte doppelblind-untersuchung: neue ansätze für die akupunktur-grundlagenforschung. Deutsche Zeitschrift für Akupunktur. 2005;48(1):18-23. 